This item was submitted to Loughborough's Research Repository by the author.

Items in Figshare are protected by copyright, with all rights reserved, unless otherwise indicated.

\title{
Structure and lithium-ion dynamics in fluoride-doped cubic Li7La3Zr2O12 (LLZO) garnet for Li solid-state battery applications
}

\section{PLEASE CITE THE PUBLISHED VERSION}

https://doi.org/10.1021/acs.jpcc.8b07704

\section{PUBLISHER}

(c) American Chemical Society

\section{VERSION}

AM (Accepted Manuscript)

\section{PUBLISHER STATEMENT}

This document is the Accepted Manuscript version of a Published Work that appeared in final form in Journal of Physical Chemistry C, copyright $\odot$ American Chemical Society after peer review and technical editing by the publisher. To access the final edited and published work see https://pubs.acs.org/doi/10.1021/acs.jpcc.8b07704.

\section{LICENCE}

CC BY-NC-ND 4.0

\section{REPOSITORY RECORD}

Yeandel, Stephen, Benjamin J. Chapman, Peter R. Slater, and Pooja Goddard. 2019. "Structure and Lithiumion Dynamics in Fluoride-doped Cubic Li7la3zr2o12 (LLZO) Garnet for Li Solid-state Battery Applications". figshare. https://hdl.handle.net/2134/36336. 


\section{THE JOURNAL OF PHYSICAL CHEMISTRY Un University}

Subscriber access provided by LOUGHBOROUGH UNIVERSITY LIBRARY

\section{C: Energy Conversion and Storage; Energy and Charge Transport}

\section{Structure and Lithium Ion Dynamics in Fluoride Doped Cubic LLZO (LiLaZrO) Garnet for Li Solid State Battery Applications}

Stephen Yeandel, Benjamin John Chapman, Peter R. Slater, and Pooja Goddard

J. Phys. Chem. C, Just Accepted Manuscript • DOI: 10.1021/acs.jpcc.8b07704 • Publication Date (Web): 10 Nov 2018

Downloaded from http://pubs.acs.org on November 14, 2018

\section{Just Accepted}

"Just Accepted" manuscripts have been peer-reviewed and accepted for publication. They are posted online prior to technical editing, formatting for publication and author proofing. The American Chemical Society provides "Just Accepted" as a service to the research community to expedite the dissemination of scientific material as soon as possible after acceptance. "Just Accepted" manuscripts appear in full in PDF format accompanied by an HTML abstract. "Just Accepted" manuscripts have been fully peer reviewed, but should not be considered the official version of record. They are citable by the Digital Object Identifier (DOI®). "Just Accepted" is an optional service offered to authors. Therefore, the "Just Accepted" Web site may not include all articles that will be published in the journal. After a manuscript is technically edited and formatted, it will be removed from the "Just Accepted" Web site and published as an ASAP article. Note that technical editing may introduce minor changes to the manuscript text and/or graphics which could affect content, and all legal disclaimers and ethical guidelines that apply to the journal pertain. ACS cannot be held responsible for errors or consequences arising from the use of information contained in these "Just Accepted" manuscripts. 


\title{
Structure and Lithium Ion Dynamics in Fluoride Doped Cubic LLZO $\left(\mathrm{Li}_{7} \mathrm{La}_{3} \mathrm{Zr}_{2} \mathrm{O}_{12}\right)$ Garnet for $\mathrm{Li}$ Solid State Battery Applications
}

\author{
Stephen R. Yeandel, ${ }^{\dagger}$ Benjamin J. Chapman, ${ }^{\dagger}$ Peter R. Slater ${ }^{\ddagger}$ and Pooja \\ Goddard (previously Panchmatia) $)^{*, \dagger}$ \\ $\dagger$ Department of Chemistry, Loughborough University, Loughborough, LE11 3TU \\ $\ddagger$ School of Chemistry, University of Birmingham, Birmingham, B15 2TT \\ E-mail: P.Goddard@lboro.ac.uk
}

\begin{abstract}
The lithium-stuffed garnet LLZO $\left(\mathrm{Li}_{7} \mathrm{La}_{3} \mathrm{Zr}_{2} \mathrm{O}_{12}\right)$, when suitably doped, is a promising candidate material for use as a solid state electrolyte within advanced Li-ion batteries. It possesses the thermal and mechanical stability of many inorganic ceramics, while exhibiting the high $\mathrm{Li}^{+}$ionic conductivities often associated with conventional liquid electrolytes, making it an ideal component for large scale energy storage. However, only the high temperature cubic phase has any meaningful Li-ion conductivity.

Typically the formation of this phase is achieved through cation doping (e.g. $\mathrm{Al}^{3+}$ on the $\mathrm{Li}$ site) to lower the $\mathrm{Li}$ content and so disrupt Li ordering. However, Li site doping, in particular, may potentially lead to some disruption of the Li ion conduction pathways and sub-optimal ionic conductivities. Consequently, other novel doping strategies involving the anion site is gaining traction, for example $\mathrm{F}^{-}$for $\mathrm{O}^{2-}$ as an alternative strategy to lower the Li content without directly blocking the lithium diffusion pathways.
\end{abstract}


For the first time, classical potentials-based simulations have been employed to simulate the incorporation of fluoride anions into LLZO. Low incorporation energies have been calculated suggesting fluoride anions are stable on the oxygen sites with a compensating lithium ion vacancy defect. Molecular dynamics (MD) calculations suggest a definitive phase transition to the more desirable cubic phase of LLZO when doped with fluoride at temperatures significantly lower than the tetragonal-cubic phase transition found for pure LLZO. Remarkably, the lithium ion transport properties are shown to improve in the fluoride doped samples particularly at low temperatures due to the stabilisation of the cubic phase, suggesting anion doping of garnet systems may be a compelling alternative route to optimise the ionic conductivity.

\section{Introduction}

Electricity generated by renewable sources such as wind or solar photovoltaics is set to revolutionise the energy industry and lead us into a more sustainable future. This eventuality is only possible provided effective solutions are devised to tackle the challenges posing the integration of renewable energy sources into the power network and automotive industry. Both applications share the need for an efficient, high energy, high power form of energy storage to support the transition from current carbon-based fossil fuels to electricity generated from more sustainable sources. ${ }^{1}$ Naturally, attention has turned towards Li-ion based systems, due to their high energy density for the solution. However, despite their dominance in small-scale portable devices, todays Li-ion batteries do not meet the necessary performance requirements demanded for large-scale applications. In order to achieve the high energy and power outputs prescribed, the Li-ion storage system must be drastically up-scaled. With this comes a significant increase to the risks associated with the safety of operation resulting from the use of conventional highly flammable organic liquid electrolytes.

Furthermore, dendritic growth caused by rapid charge/discharge cycles leads to an increased surface area at the electrode-electrolyte interface, compromising safety and lowering 
performance. ${ }^{2}$ To eliminate the risk of thermal runaway and potential rapid discharge due to a short circuit, a solid-state approach is being investigated in the design of many advanced Li-ion batteries. The high chemical, electrical and mechanical stability possessed by solid state materials make them ideal candidates for storage systems operating over wide voltage and temperature ranges. However, ultimately, the suitability of any crystalline material to act as an electrolyte hinges on its ionic conductivity. The lithium-stuffed garnet known as LLZO $\left(\mathrm{Li}_{7} \mathrm{La}_{3} \mathrm{Zr}_{2} \mathrm{O}_{12}\right)$ is one of the most promising materials for this and has attracted a great deal of attention, leading to a detailed understanding of its structure and potential lithium ion migration mechanisms. ${ }^{3}$ Considerable advances have been made over the past ten years, however there are still details of its complex structure and transport behaviour that remain unresolved.

As reported extensively in the literature, ${ }^{4-6}$ the pure garnet LLZO has the tetragonal space group $I 4_{1} /$ acd (No. 142$)^{7}$ at room temperature and is a relatively poor ionic conductor, compared to high temperature cubic garnet counterparts, with space group Ia $\overline{3} d$ (No. 230). ${ }^{8-12}$ The adoption of the tetragonal cell is a result of the high Li content, which favours $\mathrm{Li}$ ordering to limit short $\mathrm{Li}^{+}-\mathrm{Li}^{+}$interactions. Indeed the tetragonal symmetry is observed for all garnets reported so far with $7 \mathrm{Li}$ per formula unit. i.e. $\mathrm{Li}_{7} \mathrm{Ln}_{3} \mathrm{M}_{2} \mathrm{O}_{12}$ (where $\mathrm{Ln}=\mathrm{La}$, $\mathrm{Nd} ; \mathrm{M}=\mathrm{Sn}, \mathrm{Hf}, \mathrm{Zr}$ ), $\mathrm{Li}_{7} \mathrm{LnSr}_{2} \mathrm{Ta}_{2} \mathrm{O}_{12}$ (where $\left.\mathrm{Ln}=\mathrm{La}, \mathrm{Pr}, \mathrm{Nd}, \mathrm{Sm}, \mathrm{Gd}\right) .{ }^{9-12}$ 
Figure 1: Crystal structure of tetragonal $\mathrm{Li}_{7} \mathrm{La}_{3} \mathrm{Zr}_{2} \mathrm{O}_{12}{ }^{7}$ with $3 \mathrm{Li}$ coordination sites, tetrahedral $\operatorname{Li}(1)$ and octahedral $\operatorname{Li}(2)$ and $\operatorname{Li}(3)$
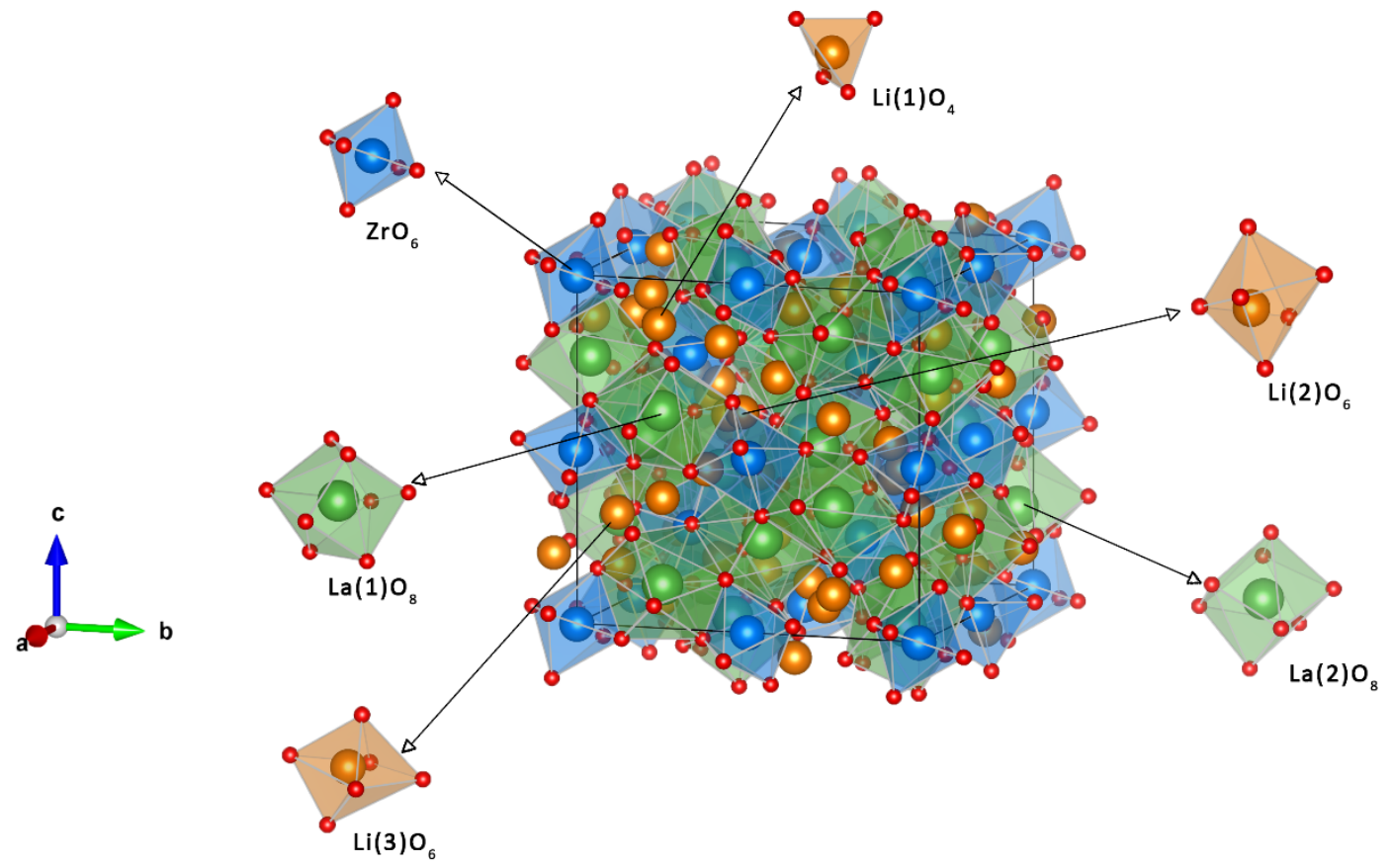

A schematic of tetragonal $\mathrm{LLZO}^{7}$ is shown in Figure 1. There are three individual fully occupied lithium sites (colored orange) within the crystal lattice: $\operatorname{Li}(1)$ is located at the tetrahedral $8 \mathrm{a}$ sites while $\operatorname{Li}(2)$ and $\operatorname{Li}(3)$ are located at the octahedral $16 \mathrm{f}$ and $32 \mathrm{~g}$ sites. Dodecahedral $\mathrm{La}(1)$ and $\mathrm{La}(2)$ are situated at the $8 \mathrm{~b}$ and 16e sites (colored green), while octahedral Zr occupies the 16c sites (colored blue). In order to convert the poorly conducting tetragonal $\mathrm{Li}_{7} \mathrm{La}_{3} \mathrm{Zr}_{2} \mathrm{O}_{12}$ into the highly conducting cubic phase at lower temperatures, doping is critical to lower the $\mathrm{Li}$ content, with the resultant $\mathrm{Li}$ vacancies disrupting the long range $\mathrm{Li}$ order. This can be achieved through $\mathrm{Nb} / \mathrm{Ta}$ doping ${ }^{7,13-15}$ on the $\mathrm{Zr}$ site, or $\mathrm{Al} / \mathrm{Ga}$ doping ${ }^{13,16,17}$ on the Li site. Indeed, Al doping can happen unintentionally simply through reaction between the garnet powders and the alumina crucibles at elevated temperatures. ${ }^{11,17,18}$ Whilst cation doping does stabilise the cubic phase at lower temperatures, impurities such as $\mathrm{CO}_{2}$ and water incorporation are well known to inhibit synthesis. Therefore novel alternative doping mechanisms are gaining research momentum, in particular anion doping strategies.

Recently, the effects of fluoride doping in lithium electrolyte materials with respect to 


\section{Methodology}

Atomistic modelling techniques are well suited to the investigation of defect and transport properties and have been applied successfully to a variety of studies on lithium battery materials. ${ }^{11,20-33}$ In the present study, potentials-based energy minimisation and molecular dynamics (MD) were employed. ${ }^{32-36}$ The advantage of employing potentials-based methods is that it allows us to study much larger systems (> 5000 species) for nanosecond timescales which would be infeasible for other methods, such as Density Functional Theory (DFT). Having said this, it is crucial to rigorously parameterise the ion-ion interactions appropriate to describe the materials in question in line with experimental observations. The GULP (General Utility Lattice Program) $\operatorname{code}^{37}$ was used for the interatomic potential verification, and within GULP the Mott-Littleton ${ }^{38}$ method was used to investigate the isolated point defects. The DL_POLY Classic 1.9 code was used for MD calculations. ${ }^{34}$

The interatomic potential model of Wang et al. ${ }^{7}$ listed in Table 1 , was used to model the pure LLZO phase. In this potential model the species are assigned a full formal charge and the shell model of Dick and Overhauser ${ }^{39}$ is employed to represent ionic polarisability. Short-range interactions were represented by the Buckingham potential ${ }^{40}$ with a cutoff of 
$12 \AA$. The short-range cutoff was reduced to $7.5 \AA$ in MD calculations to allow for faster and more efficient simulations.

Table 1: Buckingham interatomic potential parameters of Wang et al. ${ }^{7}$ used to model pure LLZO

\begin{tabular}{llll}
\hline Interaction & $A(\mathrm{eV})$ & $\rho(\AA)$ & $C\left(\mathrm{eV} . \AA^{6}\right)$ \\
\hline $\mathrm{Li}^{+}-\mathrm{O}^{2-}$ & 632.10 & 0.2906 & 0.00 \\
$\mathrm{La}^{3+}-\mathrm{O}^{2-}$ & 4579.23 & 0.3044 & 0.00 \\
$\mathrm{Zr}^{4+}-\mathrm{O}^{2-}$ & 1470.10 & 0.3500 & 0.00 \\
$\mathrm{O}^{2-}-\mathrm{O}^{2-}$ & 22764.30 & 0.1490 & 27.63 \\
\hline Species & Shell charge $(\mathrm{e})$ & Spring constant $\left(\mathrm{eV} . \AA^{-2}\right)$ & \\
\hline $\mathrm{O}^{2-}$ & -2.760 & 30.20 &
\end{tabular}

\section{Fluoride potentials}

To model fluoride doping in LLZO, additional Buckingham potential parameters are required to simulate the interaction of fluoride ions with the existing species $\left(\mathrm{Li}^{+}, \mathrm{La}^{3+}, \mathrm{Zr}^{4+}, \mathrm{O}^{2-}\right)$ in the system. In classical potentials-based modelling it is generally assumed that shortrange cation-cation interactions may be omitted due to minimal interaction of the electron clouds of the two cations, and therefore the repulsion of these species may be adequately represented by the coulombic contribution alone. Under this approximation, doping of oxide materials with cation species is relatively straightforward as only a single dopant-oxide interaction needs to be included for each dopant species. However, when the dopant is an anion, the complexity increases and new parameters for oxide-dopant, dopant-dopant and cation-dopant are required. In the case of fluoride doping of LLZO this means 5 new interactions are required; corresponding to $\mathrm{Li}^{+}-\mathrm{F}^{-}, \mathrm{La}^{3+}-\mathrm{F}^{-}, \mathrm{Zr}^{4+}-\mathrm{F}^{-}, \mathrm{F}^{-}-\mathrm{F}^{-}$and $\mathrm{O}^{2-}-\mathrm{F}^{-}$.

The majority of fluoride potential parameters were acquired from the work of Binks ${ }^{41}$ and Valerio et al. ${ }^{42}$ which have different, albeit very similar, $\mathrm{F}^{-}-\mathrm{F}^{-}$interaction parameters. Due to this difference, the compatibility and validity of these parameters must be carefully assessed. To this end, the potentials have been vetted against a combination of their bi/ternary oxides and fluorides to successfully reproduce the properties of these materials 
(see Table S4).

Despite an extensive search of the literature, a $\mathrm{Zr}^{4+}-\mathrm{F}^{-}$potential parameterisation with similar $\mathrm{F}^{-}-\mathrm{F}^{-}$potential parameters could not be located. To generate the parameters for the $\mathrm{Zr}^{4+}-\mathrm{F}^{-}$interaction a particle swarm optimisation (PSO) algorithm was employed to fit the parameters to experimental data. In general, a PSO algorithm consists of a population of fictional particles (referred to as a swarm), positioned randomly over the parameter space of the problem (for a single Buckingham potential this space is 3 dimensional, corresponding to the $A, \rho$ and $C$ parameters respectively). In this way, each particle of the swarm represents a single solution to the problem (a set of interaction parameters). The "fitness" of each particle is evaluated and then the position updated according to simple dynamics which take into account the best solution found by the particle and the best known solution across the entire swarm. In the event that a particle finds an improved solution, the best known solution for the particle is updated and, if appropriate, the best known solution of the swarm. This process is iterated and guides the swarm towards better solutions over time, whilst maintaining an exploratory behaviour. Though not guaranteed to converge to the global optimum solution, this process explores more of the potential-space than simpler optimisation algorithms which frequently converge to the nearest local minimum. Further information regarding this technique and its origins can be found in the literature. ${ }^{43-45}$

The structures of $\mathrm{LaZr}_{2} \mathrm{~F}_{11}, \mathrm{Li}_{2} \mathrm{ZrF}_{6}$ and $\mathrm{ZrF}_{4}{ }^{46-48}$ were used to fit the new $\mathrm{Zr}^{4+}-\mathrm{F}^{-}$ interaction. The use of these three structures ensures that the resultant $\mathrm{Zr}^{4+}-\mathrm{F}^{-}$potential is compatible with both the $\mathrm{Li}^{+}-\mathrm{F}^{-}$and $\mathrm{La}^{3+}-\mathrm{F}^{-}$interactions. The fitness function used was simply a weighted sum of the errors on the lattice vectors and fractional coordinates after minimisation. Thus, the weightings used in the fitness function may influence the optimisation and the resultant parameters. A number of $\mathrm{Zr}^{4+}-\mathrm{F}^{-}$Buckingham parameters were generated, corresponding to multiple different weighting schemes. A parameter set was selected which gave a good fit to the structural properties of all the three materials included in the fit, while avoiding over-fitting to one particular material. 
The $\mathrm{Zr}^{4+}-\mathrm{F}^{-}$potential was further verified by comparing the elastic constants of $\mathrm{ZrF}_{4}$ as calculated with the fitted potential, against the elastic constants obtained using DFT calculations (full details of the DFT calculation may be found in the Supporting Information). Although the elastic constants of $\mathrm{ZrF}_{4}$ were not included in the fitting of the $\mathrm{Zr}^{4+}-\mathrm{F}^{-}$ potential model, good agreement was found for the majority of elastic constants and the Bulk modulus was reproduced to within $1 \%$. The full comparison of elastic constants can be found in Tables S2 and S3.

The finalised set of fluoride potential parameters are listed in Table 2 and have been verified against multiple oxide and fluoride structures. See Supporting Information, Table S4, for full details.

Table 2: Finalised fluoride Buckingham interatomic potential parameters used in this work

\begin{tabular}{lllll}
\hline Interaction & $A(\mathrm{eV})$ & $\rho(\AA)$ & $C\left(\mathrm{eV} . \AA^{6}\right)$ & Ref. \\
\hline $\mathrm{Li}^{+}-\mathrm{F}^{-}$ & 443.83 & 0.2714 & 0.00 & $(41)$ \\
$\mathrm{La}^{3+}-\mathrm{F}^{-}$ & 2817.74 & 0.2980 & 0.00 & $(42)$ \\
$\mathrm{Zr}^{4+}-\mathrm{F}^{-}$ & 914.13 & 0.3391 & 0.00 & $\mathrm{~N} / \mathrm{A}$ \\
$\mathrm{F}^{-}-\mathrm{F}^{-}$ & 911.69 & 0.2707 & 13.80 & $(41)$ \\
$\mathrm{O}^{2-}-\mathrm{F}^{-}$ & 464.54 & 0.3362 & 22.10 & $(41)$ \\
\hline Species & Shell charge $(\mathrm{e})$ & Spring constant $\left(\mathrm{eV} . \AA^{-2}\right)$ & & \\
\hline $\mathrm{F}^{-}$ & -1.378 & 24.36 & & $(41)$
\end{tabular}

\section{Results and discussion}

\section{Structural Modelling}

The structure of tetragonal $\mathrm{LLZO}^{7}$ was found to have imaginary vibrational frequencies upon energy minimisation. These were identified to be related to the ordered lithium positions in the structure. To remove the imaginary modes a short $40 \mathrm{ps} \mathrm{MD}$ simulation at $10 \mathrm{~K}$ was performed, followed by energy minimisation.

This procedure allows the lithium ions to disorder and then relax back into new preferred positions. The final structure has only a slight change in the positions of the lithium ions, 
but the symmetry of the system has changed from tetragonal to orthorhombic. However, the deviation from tetragonal symmetry is relatively minor $(<1.2 \%)$ and all the imaginary vibrational frequencies are removed.

By examining the cubic phase of $\mathrm{LLZO}^{8}$ it becomes clear that the $\mathrm{Li}(3)$ atoms at the $32 \mathrm{~g}$ sites of tetragonal $\mathrm{LLZO}^{7}$ are at the juncture of a shallow double potential well, where both minima are partially occupied at high temperature. Thus, the procedure to remove the imaginary vibrational modes has forced the lithium atoms at the $32 \mathrm{~g}$ sites to adopt one or the other more stable configurations. Indeed, the change to orthorhombic symmetry from tetragonal is accompanied by only a $0.02 \mathrm{eV}$ per formula unit reduction in lattice energy. A comparison of the experimental and calculated lattice parameters (for both the tetragonal and orthorhombic structures of LLZO) can be found in Table S5. The pure cubic structure has been left out since it only forms at high temperatures. In light of these results, it would be interesting to experimentally study the structure of this system below room temperature to determine whether long range orthorhombic distortions are observed.

\section{Intrinsic defects}

The orthorhombic form of LLZO was used for all defect calculations as the inclusion of point defects within the tetragonal LLZO system results in an extensive long-range relaxation. This long-range relaxation in tetragonal LLZO is due to the the bulk structure relaxing to a more energetically favourable state with the introduction of a point defect (resolving the imaginary vibrational modes discussed previously); the relaxation continues further into the bulk material as the shallow double potential well is no longer symmetrical at all lithium sites.

The defect energies of several types of point defects were calculated for each species within the orthorhombic LLZO system. All point defect calculations were conducted using the Mott-Littleton method, ${ }^{38}$ using converged region sizes of $18 \AA$ and $36 \AA$ for regions I and II respectively. As the symmetry of the system has been lowered, multiple sites for each type 
of point defect were simulated. The lowest defect energy calculated for each point defect can be found in Table S7.

Using these point defect energies, the formation energy for lithium Frenkel defects as shown in Equation 1 were calculated to be $0.51 \mathrm{eV}$ comparable to other lithium conducting solids. ${ }^{11,20-33}$ The lithium Frenkel defect is of primary importance if LLZO is to be considered as a Li-ion solid state electrolyte material.

$$
\mathrm{Li}_{\mathrm{Li}}^{\mathrm{x}} \longrightarrow \mathrm{V}_{\mathrm{Li}}^{\prime}+\mathrm{Li}_{\mathrm{i}}^{\bullet}
$$

The most favoured lithium Frenkel mechanism corresponds to a lithium vacancy at the tetrahedral 8a site and the lithium interstitial sitting approximately between the lithium 8a and $32 \mathrm{~g}$ sites.

\section{Fluoride doping}

The conventional way of doping LLZO to stabilise the cubic phase and hence improve the conductivity has been to reduce the Li content through cation doping (e.g. $\mathrm{Nb}^{5+}$ on the $\mathrm{Zr}^{4+}$ site; $\mathrm{Al}^{3+}, \mathrm{Ga}^{3+}$ on the $\mathrm{Li}^{+}$site). An alternative strategy to achieve this aim would be to dope on the anion site, so that the cation network is retained. Anion doping in LLZO has seldom been considered as a means for stabilising the highly conductive cubic phase and improving the diffusion of lithium through the crystal lattice, thus the literature is limited and little is known of its performance. To date there are no published findings regarding the computational modelling of fluoride doping within the LLZO garnet and only a single experimental publication. ${ }^{4}$

Using the dopant $\mathrm{LiF}$ and a conventional solid state synthesis route, Cai et al. prepared a series of fluoride doped LLZO samples, represented by the formula $x$ wt\% LiF-LLZO $(0<x<2) .{ }^{4} \mathrm{X}$-ray diffraction analysis revealed all fluoride doped samples crystallised as the cubic garnet structure, space group $I a \overline{3} d$, whereas pure LLZO crystallised in the 
tetragonal space group $I 4_{1} a c d$. These findings suggest the incorporation of fluoride ions during synthesis is conducive towards the stabilisation of the cubic phase. In addition, conductivity of the $1.0 \mathrm{wt} \% \mathrm{LiF}-\mathrm{LLZO}$ samples was measured in the region of $5 \times 10^{-4} \mathrm{~S} / \mathrm{cm}$ at room temperature, similar to that of cubic LLZO. ${ }^{49}$ In addition, the activation energy was found to be less than that of cation doped LLZO garnets at $0.26 \mathrm{eV}$.

The purpose of fluoride doping is similar to that of previous cation dopant studies ${ }^{13,16,50,51}$ in that the resulting charged defect is compensated for by the generation of lithium vacancies. The charge compensation mechanism proposed by Cai et al. is shown in Equation 2. ${ }^{4}$

$$
\mathrm{LiF}+\mathrm{Li}_{\mathrm{Li}}^{\mathrm{x}}+\mathrm{O}_{\mathrm{O}}^{\mathrm{x}} \longrightarrow \mathrm{F}_{\mathrm{O}}^{\bullet}+\mathrm{V}_{\mathrm{Li}}^{\prime}+\mathrm{Li}_{2} \mathrm{O}
$$

This mechanism was also tested with Mott-Littleton calculations using converged region sizes of $18 \AA$ and $36 \AA$ for regions I and II respectively. Once again multiple sites were tested due to the lowering of symmetry. The lowest energy found for this mechanism was $1.9 \mathrm{eV}$. Several other mechanisms for incorporation of fluoride from LiF as well as other fluoride salts i.e. $\mathrm{LaF}_{3}$ and $\mathrm{ZrF}_{4}$, were also tested but all gave higher defect energies (Table S8). This indicates that the mechanism predicted by Cai et al. is indeed the preferred mechanism for fluoride incorporation and that the compensating defect is the formation of lithium vacancies. This level of detail is hard to observe experimentally and therefore our computational alignment with experimentally predictions is very encouraging.

The energy for forming a fluoride Frenkel has also been calculated across several sites (Equation 3).

$$
\mathrm{F}_{\mathrm{O}}^{\bullet} \longrightarrow \mathrm{V}_{\mathrm{O}}^{\bullet \bullet}+\mathrm{F}_{\mathrm{i}}^{\prime}
$$

The energy for this defect mechanism was calculated to be $4.87 \mathrm{eV}$, indicating that the fluoride ions are likely to remain on the oxygen sites, even at higher temperatures. 


\section{Phase stability}

To calculate the phase stability and lithium diffusion properties of pure and fluoride doped LLZO, classical molecular dynamics (MD) simulations were employed. All MD simulations were carried out using a 0.5 fs timestep and Nóse-Hoover thermostats/barostats ${ }^{35,36}$ within the DL_POLY Classic 1.9 code. ${ }^{34}$ Two different systems (pure and fluoride doped LLZO) were considered.

First a pure LLZO system was created, based on a $3 \times 3 \times 3$ supercell expansion of the 192 atom orthorhombic unit cell, containing a total of 5184 atoms. The second system is identical to the first but has $4 \%$ of the available oxygen sites doped with fluoride, resulting in a material with the formula $\mathrm{Li}_{6.52} \mathrm{La}_{3} \mathrm{Zr}_{2} \mathrm{O}_{11.52} \mathrm{~F}_{0.48}$. The F-doped LLZO system is roughly equivalent to the $2.0 \mathrm{wt} \%$ LiF-LLZO material in the experimental literature. ${ }^{4}$ The fluoride ions were distributed randomly across the oxygen sites and compensating lithium vacancies were likewise chosen randomly. Four random configurations were tested and the energy varied by less than $0.03 \mathrm{eV}$ per $\mathrm{Li}_{6.52} \mathrm{La}_{3} \mathrm{Zr}_{2} \mathrm{O}_{11.52} \mathrm{~F}_{0.48}$, suggesting almost no preference to the placements of $\mathrm{F}$ ions within the lattice. It must also be noted, that the lithium vacancies become mobile once temperature is introduced in MD and migrate to much more favourable positions, see SI for more details. The fluoride system is hereafter referred to as "F-doped".

The pure and F-doped systems were simulated at eight different temperatures (range 300-1000 K). At each temperature studied, the systems were equilibrated for 0.1 ns before a total of 1 ns of data were collected under an isobaric-isothermal ensemble (NPT). From these calculations the average lattice vectors were extracted and are presented as a function of simulation temperature in Figure 2. 
Figure 2: Time-averaged lattice parameters of Pure and F-doped LLZO as a function of temperature, each simulated for 1 ns using the NPT ensemble. Note that "Pure X" and "Pure Y" overlay and are equivalent

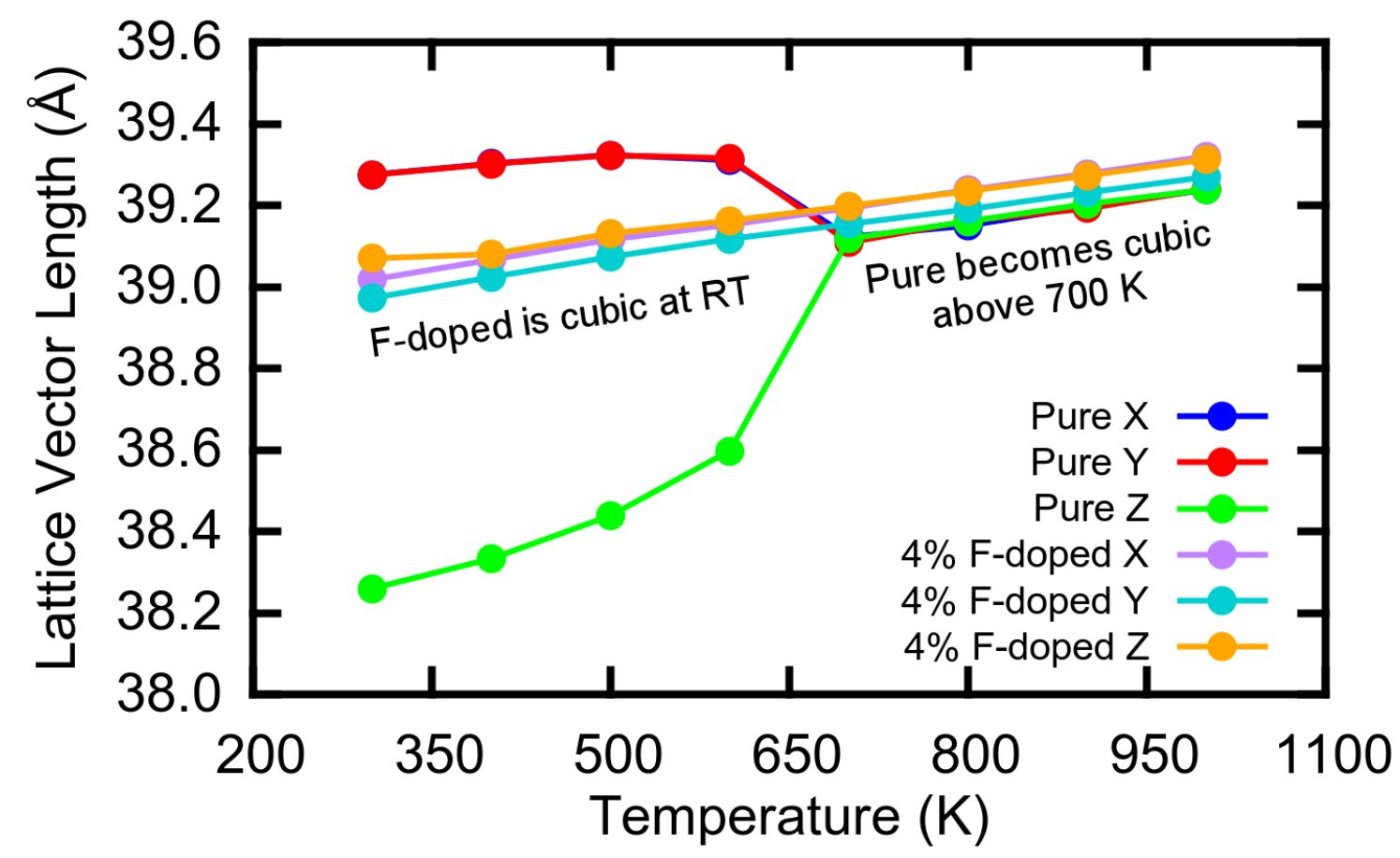

These lattice parameters clearly demonstrate that the pure LLZO system is tetragonal rather than orthorhombic between $300 \mathrm{~K}$ and $700 \mathrm{~K}$, suggesting the system is either rapidly interconverting between orthorhombic forms or that the Li site double potential well has resolved to a single minima. At $700 \mathrm{~K}$ the pure LLZO system has become cubic, somewhat in agreement with previous literature which suggests that pure tetragonal LLZO, free from humidity, undergoes a reversible phase transition to cubic symmetry at approximately 900 K. ${ }^{52,53}$ The difference in phase transition temperature is attributed to the difficulty of obtaining accurate phase transition temperatures from classical potentials-based simulations.

In comparison, the F-doped LLZO system is largely cubic with minor deviations across the entire range of temperatures studied. The slight deviations from cubic symmetry are fairly constant across all temperatures and are therefore attributed to the random distribution of fluoride defects in the system. The results are thus in strong agreement with the work of Cai et al. which suggests that LLZO cubic phase is stabilised at low temperatures due to 
incorporation of fluoride defects. ${ }^{4}$ Since the cubic phase is preferred for Li battery applications due to its higher ionic conductivity, it is critical to investigate the ionic conductivity as a result of F-doping.

\section{Lithium diffusion}

The diffusion coefficients and activation energies of pure and F-doped LLZO were also calculated using MD simulations. The average lattice parameters as obtained from the NPT calculations were kept constant. The systems were run at each temperature under a constant volume (canonical) ensemble (NVT) for a total simulation time of 2 ns at each temperature. From these simulations the mean squared displacements (MSDs) of all the ions were calculated using Equation 4, with particular interest in the $\mathrm{Li}^{+}$and $\mathrm{F}^{-}$species.

$$
\operatorname{MSD}(t)=\left\langle\left(\mathbf{r}_{i}(t)-\mathbf{r}_{i}(0)\right)^{2}\right\rangle
$$

In Equation 4, $\mathbf{r}_{i}(t)$ is the position of atom $i$ at time $t$. For lithium the MSDs are averaged over all lithium ions in the system. Additionally, a shifting time origin, $\mathbf{r}_{i}(0)$, is also employed over the full MD simulation to further improve statistics. The MSDs were found to be linear at all temperatures in both the pure and F-doped LLZO systems. An example MSD plot for the F-doped LLZO system at $1000 \mathrm{~K}$ is presented in Figure 3. 
Figure 3: MSD against time for the F-doped LLZO system at $1000 \mathrm{~K}$ using an NVT ensemble for $2 \mathrm{~ns}$

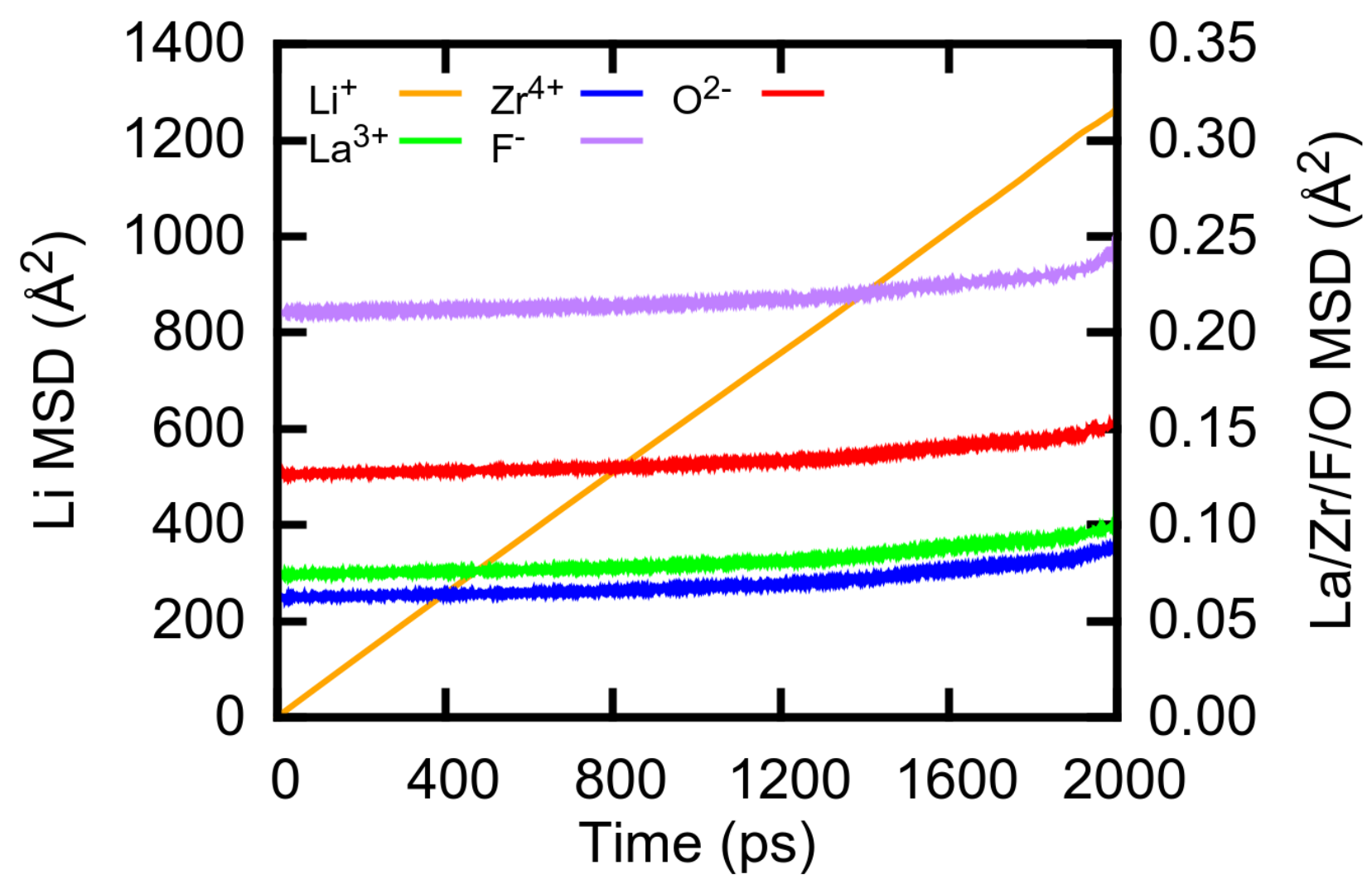

From Figure 3 it can be seen there is significant lithium diffusion at higher temperatures and that none of the other species, e.g. $\mathrm{F}^{-}$, are diffusing. The lack of $\mathrm{F}^{-}$diffusion further affirms the high fluoride Frenkel energy of $4.87 \mathrm{eV}$ (Table S8), calculated earlier using the Mott-Littleton method. The same is true at lower temperatures and also for the pure LLZO system (Table S9).

$$
\operatorname{MSD}(t)=6 D t+C
$$

Using Equation 5, the diffusion coefficients for both systems at all temperatures simulated are calculated and are listed in Table 3. 
Table 3: Diffusion coefficients $\left(\mathrm{cm}^{2} / \mathrm{s}\right)$ of pure and F-doped LLZO

\begin{tabular}{lll}
\hline Temperature (K) & pure LLZO & F-doped LLZO \\
\hline 300 & $1.69 \times 10^{-11}$ & $1.20 \times 10^{-9}$ \\
400 & $3.68 \times 10^{-10}$ & $1.19 \times 10^{-8}$ \\
500 & $1.34 \times 10^{-7}$ & $5.16 \times 10^{-7}$ \\
600 & $1.45 \times 10^{-6}$ & $1.44 \times 10^{-6}$ \\
700 & $6.72 \times 10^{-6}$ & $2.89 \times 10^{-6}$ \\
800 & $1.04 \times 10^{-5}$ & $4.91 \times 10^{-6}$ \\
900 & $1.43 \times 10^{-5}$ & $7.59 \times 10^{-6}$ \\
1000 & $1.72 \times 10^{-5}$ & $1.05 \times 10^{-5}$ \\
\hline
\end{tabular}

The F-doped LLZO system has a higher lithium diffusion coefficient for all temperatures below $600 \mathrm{~K}$. At $600 \mathrm{~K}$ the pure LLZO system is on the cusp of transitioning from tetragonal to cubic and the lithium diffusion coefficient is almost identical to F-doped LLZO at the same temperature. At $700 \mathrm{~K}$ and above (900 K experimentally ${ }^{52,53}$ ) the pure LLZO system is cubic and the lithium diffusion coefficients are now higher than in F-doped LLZO.

The lower lithium diffusion coefficients in F-doped cubic LLZO compared to pure cubic LLZO suggests that doping with fluoride ions inhibits lithium diffusion at higher temperatures $(>700 \mathrm{~K})$. The origin of the reduced diffusion coefficients at high temperatures in F-doped LLZO is likely due to the clustering of fluoride ions with lithium vacancies. Usually, the increased electronegativity of fluorine compared to oxygen would result in stronger binding of lithium ions. ${ }^{54}$ However, the defect site involved in this case is $\mathrm{F}_{\mathrm{O}}^{\bullet}$ and thus the fluoride is in effect a +1 charge state, resulting in repulsion of lithium ions and leading to clustering with lithium vacancies instead. This is supported by the computed radial distribution functions (RDFs) between lithium and oxygen and lithium and fluorine, which show a reduced probability of finding lithium in the first coordination sphere of fluorine compared to oxygen. The RDFs of Li-O and Li-F in the F-doped system at all temperatures are available in the SI.

There is however a strong argument that the creation of Li vacancies, and hence the stabilisation of the cubic phase in F-doped LLZO, is of net benefit at lower temperatures $(<$ $700 \mathrm{~K})$. Further evidence of this comes from the lithium density plots generated from the 
MD calculations, an example of which is displayed in Figure 4.

Figure 4: Schematic of lithium diffusion pathways in LLZO. Images a) and b) show pathways at $500 \mathrm{~K}$ in the pure and F-doped systems respectively. Images c) and d) show pathways at $1000 \mathrm{~K}$ in the pure and F-doped systems respectively. Zirconium octahedra (blue), fluoride ions (pink) and lithium diffusion pathways (brown) are shown, other species are omitted for clarity

\section{Pure $\quad 4 \%$ F-doped}

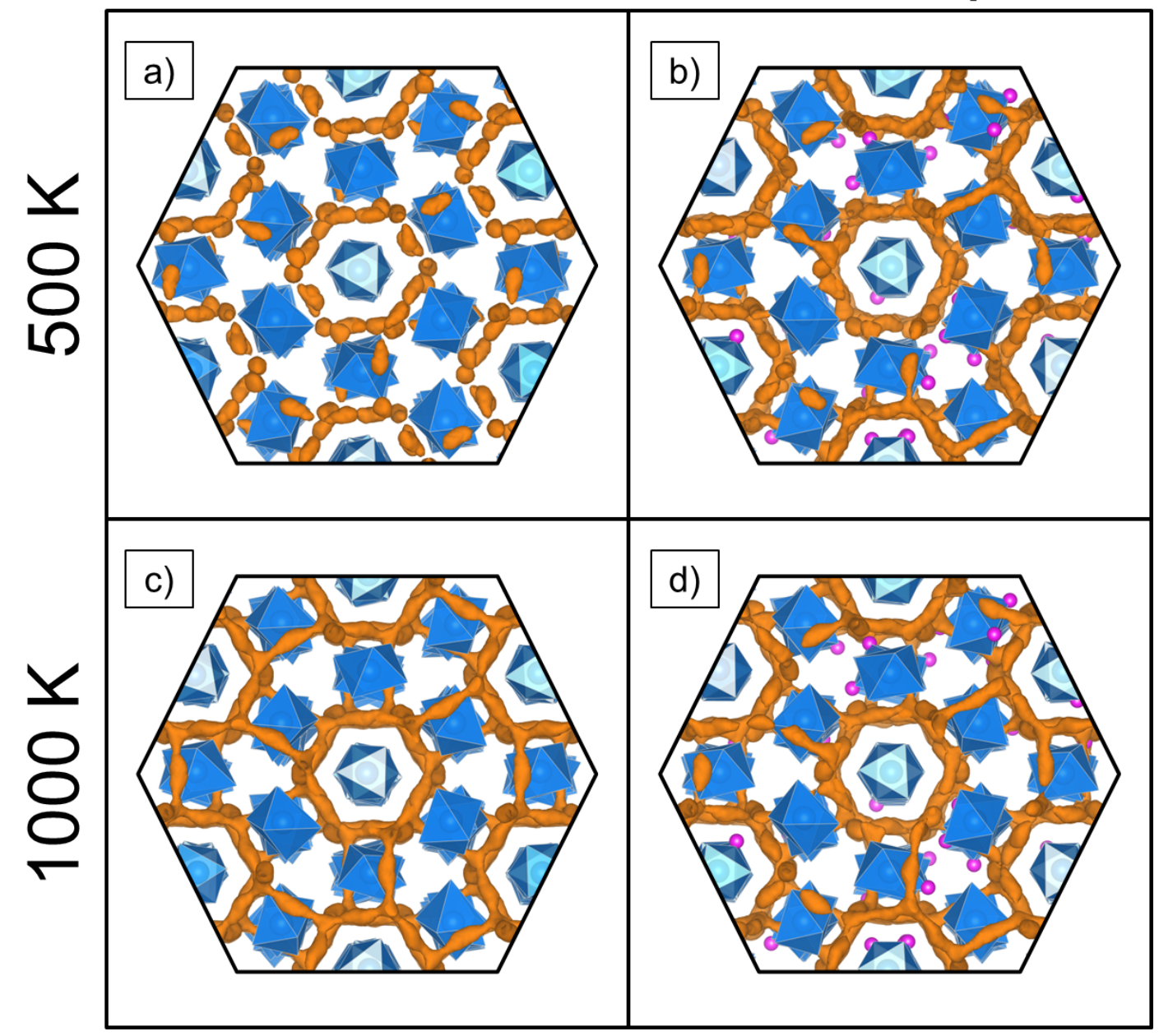

At $500 \mathrm{~K}$ the pure LLZO system displays isolated islands of lithium density, Figure 4 a), indicating infrequent hoping of lithium from one site to another. Upon doping with fluoride ions the isolated islands of lithium become more connected, indicating a more fluid movement of lithium from one site to another, Figure 4 b). At $1000 \mathrm{~K}$ the pure LLZO system has transitioned to cubic symmetry and the lithium density shows fully connected pathways, 
Figure $4 \mathrm{c}$ ), indicating that lithium ions may move freely between all 3 lithium sites. In contrast, the F-doped system at $1000 \mathrm{~K}$ also displays an increase in pathway connectivity, but to a lesser extent than in the pure system, Figure $4 \mathrm{~d}$ ). In both cases the Li migration is achieved via a vacancy hopping mechanism. These results are in agreement with the diffusion coefficient values reported in Table 3 and also suggest the incorporation of fluoride defects (and compensating lithium vacancies) leads to increased lithium diffusion at lower temperatures (compared to pure LLZO) due to the stabilisation of the cubic phase, but reduced lithium diffusion at higher temperatures due to the trapping of lithium vacancies by fluoride defects.

The trapping of lithium vacancies by fluoride defects in effect results in some degree of lithium channel blocking. However, the degree of blocking may not be as severe as when other defects, such as $\mathrm{Al}^{3+}$, are incorporated on the $\mathrm{Li}^{+}$site as there is no ion situated within the lithium channel and the lithium vacancy may not be entirely localised, allowing some degree of reduced diffusion through the channel, rather than being completely blocked. Further simulations, involving multiple defects that take into account the impact of defects on correlated motion, would be required to unravel the exact degree of blocking per-defect.

From the MSDs it is also possible to extract activation energies for lithium diffusion. An Arrhenius plot (ln $D$ versus 1000/T) for the pure and F-doped LLZO systems is presented in Figure 5. 
Figure 5: Arrhenius plot of lithium diffusion in pure and F-doped LLZO extracted using equation 6 from the 2 ns NVT MD simulation

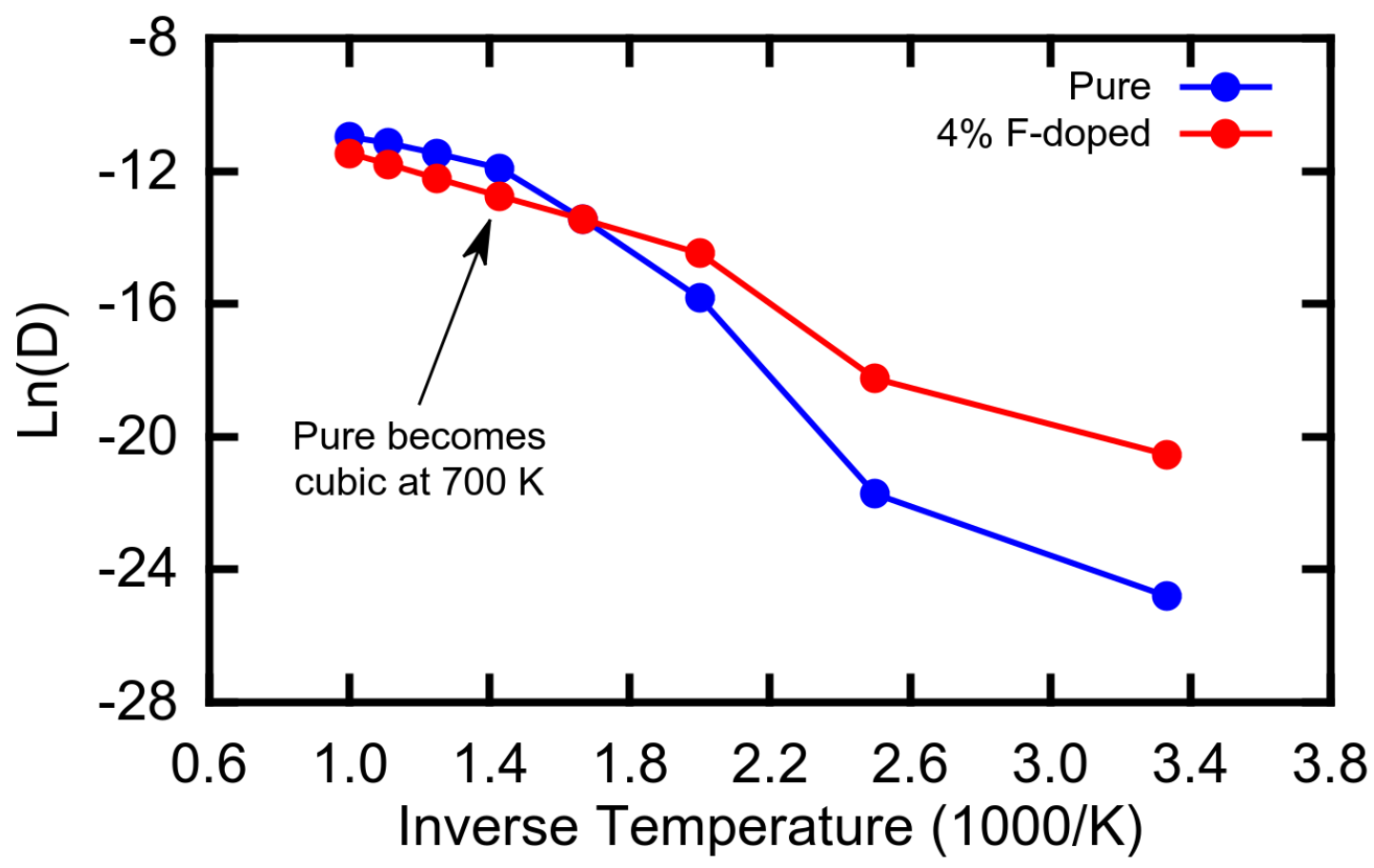

The Arrhenius plot shows good linearity for the F-doped LLZO system above $500 \mathrm{~K}$, while for the pure system the Arrhenius plot is linear only above $700 \mathrm{~K}$. This change in diffusion behaviour in the pure LLZO system once again occurs near the transition temperature of $700 \mathrm{~K}$, suggesting it is related to the tetragonal-cubic phase transition. The activation energies for lithium diffusion $\left(E_{a}\right)$ are extracted by using Equation 6 .

$$
\ln D=\frac{-E_{a}}{k_{B}} \frac{1}{T}+\ln A
$$

Thus, the gradient of the Arrhenius plot (Figure 5) is proportional to the Li migration barrier. The activation energy for species other than $\mathrm{Li}^{+}$have not been calculated as they display no diffusion, even at elevated temperatures, however the MSD have been shown in the supplementary information at all temperatures, Table S9. Pure LLZO undergoes a phase transition at approximately $700 \mathrm{~K}$, and thus two activation energies are calculated, one for each phase. Only a single activation energy is calculated for F-doped LLZO as the system 
remains cubic at all temperatures. The diffusion coefficients below $500 \mathrm{~K}$ have not been used in the calculation of the activation energies in both pure and F-doped LLZO as they have not reached the diffusive regime. The activation energies for lithium ion diffusion are presented in Table 4.

Table 4: Calculated migration barriers for lithium diffusion in pure and F-doped LLZO. The migration barriers are calculated over two temperature ranges for both materials to facilitate easier comparison. The results clearly show enhanced diffusion at lower temperatures in the F-doped system

\begin{tabular}{llll}
\hline System & Crystal system & Temperature range & $E_{a}(\mathrm{eV})$ \\
\hline \multirow{2}{*}{ Pure } & Tetragonal & $500-700$ & 0.59 \\
& Cubic & $700-1000$ & 0.19 \\
\hline \multirow{2}{*}{ F-doped } & Cubic & $500-1000$ & 0.26 \\
\hline
\end{tabular}

The activation energies for lithium ion diffusion show fairly good agreement with literature values of $0.41-0.50 \mathrm{eV}$ for pure tetragonal LLZO ${ }^{55-57}$ (calculated $0.59 \mathrm{eV}$ ) and 0.30$0.37 \mathrm{eV}$ for pure cubic LLZO ${ }^{49,58}$ (calculated $0.19 \mathrm{eV}$ ). The calculated activation energies are comparable to those found for cation doped LLZO, namely Al (0.26-0.36 eV), ${ }^{16,59} \mathrm{Ta}$ $(0.22-0.35 \mathrm{eV})^{13,60}$ and $\mathrm{Nb}(0.47 \mathrm{eV}) .{ }^{15}$

Additionally, the calculated activation energy in F-doped LLZO of $0.26 \mathrm{eV}$ is in excellent agreement with the experimental value of $0.26 \mathrm{eV}$ obtained by Cai et al. for the $1.0 \mathrm{wt} \%$ sample. ${ }^{4}$ Furthermore, Figure 5 shows that the highly conducting regime in F-doped LLZO continues down to $500 \mathrm{~K}$, whereas in pure LLZO the highly conducting regime is observed only above $700 \mathrm{~K}$.

The agreement with experiment is further bolstered by the experimentally observed improvement in the sinterability of F-doped LLZO during synthesis, ${ }^{4}$ which will act to reduce the effect of grain boundaries on the experimental activation energies. The increased sinterability therefore allows a better comparison with simulations which omit such extended defects. 


\section{Conclusions}

The aim of this investigation was to use computational modelling to better understand the local structure and Li ion dynamics upon fluorine doping in Li stuffed LLZO garnet material. Fluoride doping is an interesting novel alternative doping strategy to stabilise the highly conductive cubic phase at lower temperatures. In order to do this an extension to the potential model of Wang et al. ${ }^{7}$ has been developed which allows for the incorporation of fluoride ion defects into the lithium-stuffed garnet LLZO. The Buckingham potential model has been generated using known parameters found in the literature ${ }^{41,42}$ alongside new parameters for the $\mathrm{Zr}^{4+}-\mathrm{F}^{-}$interaction derived using a Particle Swarm Optimisation (PSO) algorithm. ${ }^{43-45}$

This development allowed us to simulate 5000+ atom systems over a range of temperatures on nanosecond timescales using classical MD techniques. The results demonstrate a correlation between the phase transitions on $\mathrm{F}^{-}$doping and the enhance Li ion dynamics at lower temperatures. From our calculations we conclude that,

1) doping the lithium-stuffed garnet LLZO with LiF has a defect energy of $1.90 \mathrm{eV}$, with the generation of lithium vacancies as the compensating mechanism in line with prediction from experimental reports, ${ }^{4}$

2) the F-doped LLZO system prefers to be cubic at much lower temperatures ( 500 K) than the pure LLZO systems and

3) the lithium diffusion in these low temperature cubic phases of F-doped LLZO is comparable to pure cubic or cation doped LLZO, making the anion doping mechanism a competitive alternative to cation doping which introduces other complexities such as sensitivity to humidity.

Our modelling results confirm that fluoride incorporation is an attractive and viable alternative route to obtaining highly conducting cubic LLZO at low temperatures. Furthermore, our approach demonstrates the feasibility of studying anionic doping using potential models, which may now be applied to other materials. Future work will therefore extend towards 
studying the effects of other anion defects such as carbonate and hydroxide defects.

\section{Acknowledgement}

PG and SRY acknowledge the support of EPSRC SUPERGEN grant, EP/N001982/1. PRS acknowledges the support of EPSRC grant EP/R024006/1: ICSF Wave 1: GENESIS: Garnet Electrolytes for New Energy Storage Integrated Solutions This paper recognises the use of the "Hydra" High Performance System at Loughborough University. Via our membership of the UK's HEC Materials Chemistry Consortium, which is funded by EPSRC (EP/L000202), this work used the ARCHER UK National Supercomputing Service (http://www.archer.ac.uk). Furthermore, PG and SRY acknowledge the help of the ScienceIT team at Loughborough.

\section{Supporting Information Available}

Information on the potential model used for this work, including calculated and experimental comparisons of the crystal structures used to fit and validate the potential model, point defect energies and all the doping mechanisms are available in the Supporting Information. Furthermore, MSD data at all temperatures for both pure and F-doped LLZO have also been included.

This material is available free of charge via the Internet at http://pubs.acs.org/.

\section{References}

(1) Moriarty, P.; Honnery, D. What is the global potential for renewable energy? Renewable and Sustainable Energy Reviews 2012, 16, 244-252.

(2) Li, Z.; Huang, J.; Liaw, B. Y.; Metzler, V.; Zhang, J. A review of lithium deposition 
in lithium-ion and lithium metal secondary batteries. Journal of Power Sources 2014, 254, 168-182.

(3) Cussen, E. J. Structure and ionic conductivity in lithium garnets. Journal of Materials Chemistry 2010, 20, 5167-5173.

(4) Cai, L.; Zhao-Yin, W.; Kun, R. High ion conductivity in garnet-type F-doped Li (7) La (3) Zr (2) O12. Journal of Inorganic Materials 2015, 30, 995-1000.

(5) Awaka, J.; Kijima, N.; Hayakawa, H.; Akimoto, J. Synthesis and structure analysis of tetragonal Li 7 La 3 Zr 2 O 12 with the garnet-related type structure. Journal of Solid State Chemistry 2009, 182, 2046-2052.

(6) Thangadurai, V.; Narayanan, S.; Pinzaru, D. Garnet-type solid-state fast Li ion conductors for Li batteries: critical review. Chemical Society Reviews 2014, 43, 4714-4727.

(7) Wang, Y.; Huq, A.; Lai, W. Insight into lithium distribution in lithium-stuffed garnet oxides through neutron diffraction and atomistic simulation: Li 7-x La 3 Zr 2-x Ta x O $12(\mathrm{x}=0-2)$ series. Solid State Ionics 2014, 255, 39-49.

(8) Awaka, J.; Takashima, A.; Kataoka, K.; Kijima, N.; Idemoto, Y.; Akimoto, J. Crystal structure of fast lithium-ion-conducting cubic Li7La3Zr2O12. Chemistry Letters 2010, 40, 60-62.

(9) Percival, J.; Kendrick, E.; Smith, R.; Slater, P. Cation ordering in Li containing garnets: synthesis and structural characterisation of the tetragonal system, Li 7 La 3 Sn 2 O 12. Dalton Transactions 2009, 5177-5181.

(10) Howard, M.; Clemens, O.; Parvathy, A.; Anderson, P.; Slater, P. Synthesis and ionic conductivity of new high Li ion content garnets, LnSr 2 Ta 2 Li 7 O 12 (Ln= La, Pr, Nd, Sm, Gd). Journal of Alloys and Compounds 2016, 670, 78-84. 
(11) Howard, M.; Clemens, O.; Knight, K.; Anderson, P.; Hafiz, S.; Panchmatia, P. M.; Slater, P. Synthesis, conductivity and structural aspects of Nd 3 Zr 2 Li 7- 3x Al x O 12. Journal of Materials Chemistry A 2013, 1, 14013-14022.

(12) Awaka, J.; Kijima, N.; Kataoka, K.; Hayakawa, H.; Ohshima, K.-i.; Akimoto, J. Neutron powder diffraction study of tetragonal Li 7 La 3 Hf 2 O 12 with the garnet-related type structure. Journal of Solid State Chemistry 2010, 183, 180-185.

(13) Allen, J. L.; Wolfenstine, J.; Rangasamy, E.; Sakamoto, J. Effect of substitution (Ta, Al, Ga) on the conductivity of Li 7 La 3 Zr 2 O 12. Journal of Power Sources 2012, 206, 315-319.

(14) Liu, C.; Rui, K.; Shen, C.; Badding, M. E.; Zhang, G.; Wen, Z. Reversible ion exchange and structural stability of garnet-type Nb-doped Li7La3Zr2O12 in water for applications in lithium batteries. Journal of Power Sources 2015, 282, 286-293.

(15) Ohta, S.; Kobayashi, T.; Asaoka, T. High lithium ionic conductivity in the garnet-type oxide Li7- X La3 (Zr2- X, NbX) O12 (X=0-2). Journal of Power Sources 2011, 196, $3342-3345$.

(16) Rangasamy, E.; Wolfenstine, J.; Sakamoto, J. The role of Al and Li concentration on the formation of cubic garnet solid electrolyte of nominal composition Li 7 La 3 Zr 2 O 12. Solid State Ionics 2012, 206, 28-32.

(17) Howard, M.; Clemens, O.; Kendrick, E.; Knight, K.; Apperley, D.; Anderson, P.; Slater, P. Effect of Ga incorporation on the structure and Li ion conductivity of La 3 Zr 2 Li 7 O 12. Dalton Transactions 2012, 41, 12048-12053.

(18) Shin, D. O.; Oh, K.; Kim, K. M.; Park, K.-Y.; Lee, B.; Lee, Y.-G.; Kang, K. Synergistic multi-doping effects on the Li7La3Zr2O12 solid electrolyte for fast lithium ion conduction. Scientific reports 2015, 5 . 
(19) Li, Y.; Zhou, W.; Xin, S.; Li, S.; Zhu, J.; Lü, X.; Cui, Z.; Jia, Q.; Zhou, J.; Zhao, Y. et al. Fluorine-doped antiperovskite electrolyte for all-solid-state lithium-ion batteries. Angewandte Chemie International Edition 2016, 55, 9965-9968.

(20) Panchmatia, P. M.; Armstrong, A. R.; Bruce, P. G.; Islam, M. S. Lithium-ion diffusion mechanisms in the battery anode material Li $1+\mathrm{x}$ V 1- x O 2. Physical Chemistry Chemical Physics 2014, 16, 21114-21118.

(21) Armstrong, A. R.; Lyness, C.; Panchmatia, P. M.; Islam, M. S.; Bruce, P. G. The lithium intercalation process in the low-voltage lithium battery anode Li1 + xV1- xO2. Nature materials 2011, 10, 223-229.

(22) Andreev, Y. G.; Panchmatia, P. M.; Liu, Z.; Parker, S. C.; Islam, M. S.; Bruce, P. G. The shape of TiO2-B nanoparticles. Journal of the American Chemical Society 2014, 136, 6306-6312.

(23) Fisher, C. A.; Islam, M. S. Surface structures and crystal morphologies of LiFePO 4: relevance to electrochemical behaviour. Journal of Materials Chemistry 2008, 18, $1209-1215$.

(24) Schroeder, M.; Eames, C.; Tompsett, D. A.; Lieser, G.; Islam, M. S. Li x FeF 6 (x=2, 3, 4) battery materials: structural, electronic and lithium diffusion properties. Physical Chemistry Chemical Physics 2013, 15, 20473-20479.

(25) Islam, M. S.; Driscoll, D. J.; Fisher, C. A.; Slater, P. R. Atomic-scale investigation of defects, dopants, and lithium transport in the LiFePO4 olivine-type battery material. Chemistry of Materials 2005, 17, 5085-5092.

(26) Gardiner, G. R.; Islam, M. S. Anti-site defects and ion migration in the LiFe0. 5Mn0. 5PO4 mixed-metal cathode material. Chemistry of Materials 2009, 22, 1242-1248. 
(27) Kuganathan, N.; Islam, M. Li2MnSiO4 lithium battery material: atomic-scale study of defects, lithium mobility, and trivalent dopants. Chemistry of materials 2009, 21, 5196-5202.

(28) Lee, S.; Park, S. S. Atomistic simulation study of mixed-metal oxide (LiNi1/3Co1/3Mn1/3O2) cathode material for lithium ion battery. The Journal of Physical Chemistry C 2012, 116, 6484-6489.

(29) Tealdi, C.; Spreafico, C.; Mustarelli, P. Lithium diffusion in Li 1- x FePO 4: the effect of cationic disorder. Journal of Materials Chemistry 2012, 22, 24870-24876.

(30) Adams, S.; Rao, R. P. Simulated defect and interface engineering for high power Li electrode materials. Solid State Ionics 2011, 184, 57-61.

(31) Salanne, M.; Marrocchelli, D.; Watson, G. W. Cooperative mechanism for the diffusion of Li+ ions in LiMgSO4F. The Journal of Physical Chemistry C 2012, 116, 1861818625.

(32) Catlow, C. R. A. Computer modeling in inorganic crystallography; Academic Press, 1997.

(33) Islam, M. S.; Fisher, C. A. Lithium and sodium battery cathode materials: computational insights into voltage, diffusion and nanostructural properties. Chemical Society Reviews 2014, 43, 185-204.

(34) Smith, W.; Yong, C.; Rodger, P. DL_POLY: Application to molecular simulation. Molecular Simulation 2002, 28, 385-471.

(35) Nosé, S. A unified formulation of the constant temperature molecular dynamics methods. The Journal of chemical physics 1984, 81, 511-519.

(36) Hoover, W. G. Canonical dynamics: equilibrium phase-space distributions. Physical review $A$ 1985, 31, 1695. 
(37) Gale, J. D. GULP: A computer program for the symmetry-adapted simulation of solids. Journal of the Chemical Society, Faraday Transactions 1997, 93, 629-637.

(38) Mott, N.; Littleton, M. Conduction in polar crystals. I. Electrolytic conduction in solid salts. Transactions of the Faraday Society 1938, 34, 485-499.

(39) Dick Jr, B.; Overhauser, A. Theory of the dielectric constants of alkali halide crystals. Physical Review 1958, 112, 90.

(40) Buckingham, R. A. The classical equation of state of gaseous helium, neon and argon. Proceedings of the Royal Society of London A: Mathematical, Physical and Engineering Sciences. 1938; pp 264-283.

(41) Binks, D. J. Computational modelling of zinc oxide and related oxide ceramics. Ph.D. thesis, University of Surrey, 1994.

(42) Valerio, M.; Jackson, R.; De Lima, J. Derivation of potentials for the rare-earth fluorides, and the calculation of lattice and intrinsic defect properties. Journal of Physics: Condensed Matter 2000, 12, 7727.

(43) Kennedy, J.; Eberhart, R. Particle swarm optimization, proceedings of IEEE International Conference on neural networks (ICNN95) in. 1995.

(44) Shi, Y.; Eberhart, R. A modified particle swarm optimizer. Evolutionary Computation Proceedings, 1998. IEEE World Congress on Computational Intelligence., The 1998 IEEE International Conference on. 1998; pp 69-73.

(45) González, D.; Davis, S. Fitting of interatomic potentials without forces: A parallel particle swarm optimization algorithm. Computer Physics Communications 2014, 185, 3090-3093.

(46) Martineau, C.; Legein, C.; Body, M.; Péron, O.; Boulard, B.; Fayon, F. Structural 
investigation of $\alpha$-LaZr 2 F 11 by coupling X-ray powder diffraction, $19 \mathrm{~F}$ solid state NMR and DFT calculations. Journal of Solid State Chemistry 2013, 199, 326-333.

(47) Brunton, G. Li2ZrF6. Acta Crystallographica Section B: Structural Crystallography and Crystal Chemistry 1973, 29, 2294-2296.

(48) Papiernik, R.; Mercurio, D.; Frit, B. Structure du tétrafluorure de zirconium, ZrF4 $\alpha$. Acta Crystallographica Section B: Structural Crystallography and Crystal Chemistry 1982, 38, 2347-2353.

(49) Murugan, R.; Thangadurai, V.; Weppner, W. Fast lithium ion conduction in garnettype Li7La3Zr2O12. Angewandte Chemie International Edition 2007, 46, 7778-7781.

(50) Wolfenstine, J.; Ratchford, J.; Rangasamy, E.; Sakamoto, J.; Allen, J. L. Synthesis and high Li-ion conductivity of Ga-stabilized cubic Li 7 La 3 Zr 2 O 12. Materials Chemistry and Physics 2012, 134, 571-575.

(51) Miara, L. J.; Richards, W. D.; Wang, Y. E.; Ceder, G. First-principles studies on cation dopants and electrolyte- cathode interphases for lithium garnets. Chemistry of Materials 2015, 27, 4040-4047.

(52) Larraz, G.; Orera, A.; Sanjuan, M. Cubic phases of garnet-type Li 7 La 3 Zr 2 O 12: the role of hydration. Journal of Materials Chemistry A 2013, 1, 11419-11428.

(53) Chen, Y.; Rangasamy, E.; dela Cruz, C. R.; Liang, C.; An, K. A study of suppressed formation of low-conductivity phases in doped Li 7 La 3 Zr 2 O 12 garnets by in situ neutron diffraction. Journal of Materials Chemistry A 2015, 3, 22868-22876.

(54) Wang, Z.; Shao, G. Theoretical design of solid electrolytes with superb ionic conductivity: alloying effect on $\mathrm{Li}+$ transportation in cubic Li 6 PA 5 X chalcogenides. Journal of Materials Chemistry A 2017, 5, 21846-21857. 
(55) Wolfenstine, J.; Rangasamy, E.; Allen, J. L.; Sakamoto, J. High conductivity of dense tetragonal Li 7 La 3 Zr 2 O 12. Journal of Power Sources 2012, 208, 193-196.

(56) Buschmann, H.; Dölle, J.; Berendts, S.; Kuhn, A.; Bottke, P.; Wilkening, M.; Heitjans, P.; Senyshyn, A.; Ehrenberg, H.; Lotnyk, A. et al. Structure and dynamics of the fast lithium ion conductor Li 7 La 3 Zr 2 O 12. Physical Chemistry Chemical Physics 2011, 13, 19378-19392.

(57) Loho, C.; Djenadic, R.; Bruns, M.; Clemens, O.; Hahn, H. Garnet-type Li7La3Zr2O12 solid electrolyte thin films grown by CO2-laser assisted CVD for all-solid-state batteries. Journal of The Electrochemical Society 2017, 164, A6131-A6139.

(58) Shimonishi, Y.; Toda, A.; Zhang, T.; Hirano, A.; Imanishi, N.; Yamamoto, O.; Takeda, Y. Synthesis of garnet-type Li 7- x La 3 Zr 2 O 12- 1/2x and its stability in aqueous solutions. Solid State Ionics 2011, 183, 48-53.

(59) Chen, R.-J.; Huang, M.; Huang, W.-Z.; Shen, Y.; Lin, Y.-H.; Nan, C.-W. Effect of calcining and Al doping on structure and conductivity of Li7La3Zr2O12. Solid State Ionics 2014, 265, 7-12.

(60) Li, Y.; Han, J.-T.; Wang, C.-A.; Xie, H.; Goodenough, J. B. Optimizing Li+ conductivity in a garnet framework. Journal of Materials Chemistry 2012, 22, 15357-15361. 


\section{TOC Graphic}

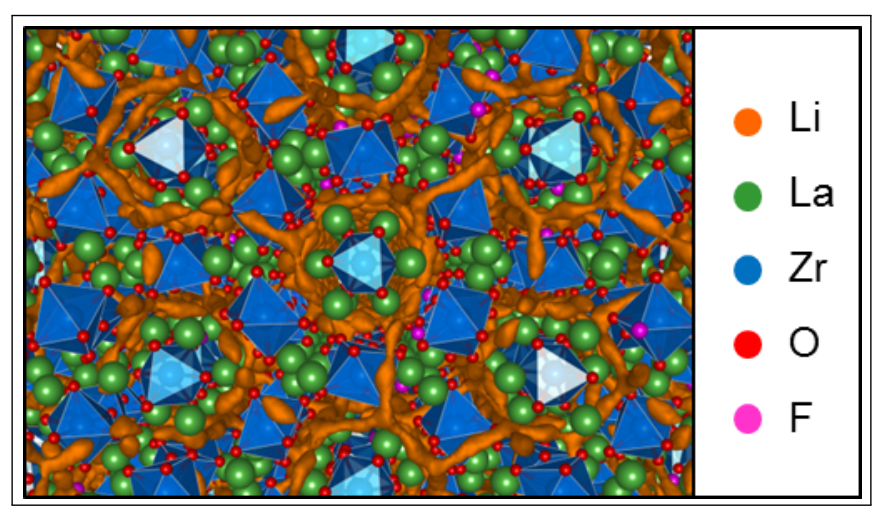




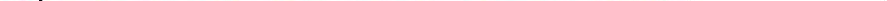

